\title{
Identification of $M$ s2, a Novel Locus Controlling Male-fertility Restoration of Cytoplasmic Male- sterility in Onion (Allium Cepa L.), and Development of Tightly Linked Molecular Markers
}

Nari Yu

Chonnam National University

Sunggil Kim ( $\nabla$ dronion@jnu.ac.kr)

Chonnam National University https://orcid.org/0000-0001-8555-2995

\section{Research Article}

Keywords: Onion (Allium cepa L.), Cytoplasmic male-sterility, Restorer-of-fertility gene, Bulked segregant analysis, RNA-seq

Posted Date: June 29th, 2021

DOl: https://doi.org/10.21203/rs.3.rs-643567/v1

License: (c) (1) This work is licensed under a Creative Commons Attribution 4.0 International License. Read Full License 


\section{Abstract}

Cytoplasmic male-sterility (CMS) has been exclusively used to produce $\mathrm{F}_{1}$ hybrid seeds of onion (Allium cepa L.). A single nuclear locus, $M s$, is known to restore male-fertility of CMS in onions. Unstable malesterile onions producing a small amount of pollen grains have been identified in a previous study. When such unstable male-sterile onions were crossed with stable male-sterile onions containing CMS-T cytoplasm, male-fertility was completely restored, although genotypes of the Ms locus were homozygous recessive. Inheritance patterns indicated that male-fertility restoration was controlled by a single locus designated as Ms2. A combined approach of bulked segregant analysis and RNA-seq was used to identify candidate genes for the Ms2 locus. High resolution melting (HRM) markers were developed based on single nucleotide polymorphisms (SNPs) detected by RNA-Seq. Comparative mapping of the Ms2 locus showed that Ms2 was positioned at the end of chromosome 2 with a distance of approximately $70 \mathrm{cM}$ away from the Ms locus. Although 38 contigs containing reliable SNPs were analyzed using recombinants selected from 1,344 individuals, no contig showed perfect linkage to Ms2. Interestingly, transcription levels of orf725, a CMS-associated gene in onions, were significantly reduced in male-fertile individuals of segregating populations. However, no significant change in its transcription level was observed in individuals of a segregating population with male-fertility phenotypes determined by the Ms locus, suggesting that male-fertility restoration mechanism of $M s 2$ might be different from that of the Ms locus.

\section{Introduction}

Male-sterility is an inability of producing functional pollen grains in plants. Any critical defects from initial stages of male gametophyte development to anther dehiscence can result in male-sterility (Wan et al. 2019). Gynodioecy attributed by male-sterility is a widespread reproductive strategy in flowering plants. Approximately $7 \%$ of angiosperm species are known to show gynodioecy (McCauley and Bailey 2009). Depending on genomic positions of causal genes, male-sterility is classified as genic male-sterility (GMS) and cytoplasmic male-sterility (CMS). Genes inducing GMS and CMS are located in nuclear and mitochondrial genomes, respectively (Chen and Liu 2013).

CMS is caused by abnormal mitochondrial genes and thus is mostly maternally inherited in plants. Plant mitochondrial genomes show exceptional variations of size and structure (Chen et al. 2017). In contrast to small and circular animal mitogenomes (14-20 kb), plant mitogenomes vary in size from $66 \mathrm{~kb}$ for Viscum scurruloideum (Skippington et al. 2015) to $11.3 \mathrm{Mb}$ for Silene conica (Sloan et al. 2012). Size variations occur even in the same species (Sloan et al. 2010). Complexity of genome structures is another feature of plant mitogenomes. Linear, circular, and branched forms of genome structures have been reported (Backert et al. 1997; Oldenburg and Bendich 2001; Sloan 2013).

Multipartite structures and sublimons are generally observed in plants. They make plant mitogenomes even more complex. A master circle of plant mitogenomes is generally divided into multipartite of subcircles. Besides a mater circle, sublimons present at low copy numbers have been reported in many 
plant species (Fauron et al. 1995; Satoh et al. 2004; Allen et al. 2007; Kitazaki and Kubo 2010; Chen et al. 2017). Abundant repeat sequences distributed in plant mitogenomes are known to be responsible for dynamic rearrangements of plant mitogenomes (Small et al. 1989; Albert et al. 1998; Kmiec et al. 2006; Woloszynska and Trojanowski 2009). CMS-associated genes cloned so far are mostly created by repeatmediated mtDNA rearrangements. Frequently, CMS-associated genes are chimeric genes composed of partial sequences of known mitochondrial genes and unknown sequences (Hanson and Bentolila 2004; Chen and Liu 2013; Chen et al. 2017).

Sublimons present at a substoichiometric level sometimes become sources of new variations (Sakai and Imamura 1993; Bellaoui et al. 1998; Janska et al. 1998; Arrieta-Montiel et al. 2001). Copy number of sublimons can be infrequently increased by a substoichiometric shifting (SSS) mechanism (Bellaoui et al. 1998; Janska et al. 1998; Sandhu et al. 2007). Although the origin and maintenance of sublimons are not clearly resolved yet, tissue culture (Kanazawa et al., 1994) and nuclear genes such as Msh1 (Abdelnoor et al. 2003), OSB1 (Zaegel et al. 2006), and RecA (Shedge et al. 2007) are known to trigger SSS. These nuclear genes are likely to control specific stoichiometry of sublimons throughout generations. CMS phenotypes can appear by SSS of sublimons harboring CMS-associated genes (ArrietaMontiel et al. 2001; Sandhu et al. 2007).

Meanwhile, male-sterility caused by CMS-associated genes can be suppressed by nuclear restorer-offertility (Rf) genes. Majority of Rf genes isolated so far encode pentatricopeptide repeat (PPR) proteins (Gaborieau et al. 2016). PPR-coding genes consist of a large family in plants. Most of them are known to regulate gene expression in organelles at the transcription level (Barkan and Small 2014; Manna 2015). In addition, Rf genes encoding other proteins such as aldehyde dehydrogenase (Cui et al. 1996), putative acyl-carrier proteins (Fujii and Toriyama 2009), glycine-rich proteins (Hu et al. 2012), and peptidases (Kitazaki et al. 2015) have been reported.

CMS is an ideal topic for studying commutation between nucleus and mitochondrion. It has also been utilized in commercial production of $\mathrm{F}_{1}$ hybrid seeds in many crops (Bohra et al. 2016). In the case of onion, $\mathrm{CMS}$ is a unique emasculation tool for $\mathrm{F}_{1}$ hybrid breeding. Two major types of $\mathrm{CMS}$ have been reported in onions. A male-sterile (MS) onion named as CMS-S was first discovered by Jones and Emsweller (1936). Inheritance patterns indicated that male-fertility of CMS-S could be restored by a single Rf locus designated as Ms (Jones and Clarke 1943). Another type of CMS called CMS-T was reported by Berninger (1965). Three independent Rf loci have been assumed to be involved in male-fertility restoration (Schweisguth 1973).

A chimeric mitochondrial gene, orf725, composed of partial sequences of coxl and unknown sequences has been suggested as a CMS-associated gene in onions (Kim et al. 2009). Comparative analysis of complete mitogenomes of normal, CMS-S, and CMS-T supports a role of orf725 in CMS induction. orf725 was present in both CMS-S and CMS-T cytoplasm (Kim et al. 2016, 2019b). In conflict with Schweisguth (1973), our previous study (Kim 2014) has shown that male-fertility of both CMS-S and CMS-T can be restored by the common Ms locus. Many molecular markers tagging the Ms locus have been developed 
(Gökçe and Havey 2002; Bang et al. 2013; Park et al. 2013; Yang et al. 2013; Havey 2013; Kim 2014; Huo et al. 2015; Kim et al. 2015a). Some of them show perfect linkage disequilibrium with the Ms locus (Kim 2014; Huo et al. 2015; Kim et al. 2015a).

In addition to the Ms locus, a novel Rf locus was identified in this study from onion populations in which the Ms locus was fixed as a homozygous recessive genotype. A combined approach of bulked segregant analysis (BSA, Michelmore et al. 1991) and RNA-seq was used to develop tightly linked molecular markers and to determine the chromosomal location of this novel Rf locus. Interaction between $\mathrm{Ms}$ and the novel loci and the mechanism of male-fertility restoration by the novel Rf locus were also discussed in this study.

\section{Materials And Methods}

\section{Plant materials}

Onions showing unstable MS phenotypes in our previous study (Kim et al. 2019a) were used. Two of them in the $\mathrm{OP}_{2}$ population were crossed with onions showing stable MS phenotypes and containing CMS-T cytoplasm. Two resulting populations were designated as TUMS4 and TUMS9, respectively. Malefertile (MF) plants in these two populations were self-pollinated or cross-pollinated with MS plants in the same populations to produce large-sized segregating populations. A detail pedigree of onion populations used in this study is depicted in Supplementary Fig. 1.

Seedlings germinated in 128-cell plug trays were transplanted into pots in the greenhouse or fields of

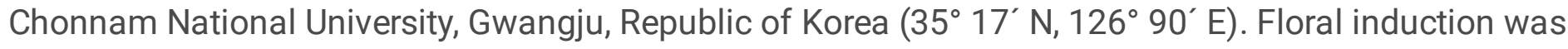
initiated naturally during winter seasons. Male-fertility phenotypes were determined by visual examination in June for three years (2018-2020). Statistics of temperatures during flowering seasons are summarized in Supplementary Table 1. Morphologies of anthers and pollen grains were examined using a dissecting microscope (Stemi 2000-C; ZEISS, Oberkochen, Germany) and a scanning electron microscope (SEM, JSM-IT300; JEOL, Tokyo, Japan), respectively.

\section{RNA extraction, RNA-Seq analysis, and real-time RT-PCR}

To perform a combined analysis of BSA and RNA-seq, flower buds of $10 \mathrm{MF}$ and $10 \mathrm{MS}$ individuals of the TUMS4 population were pooled, respectively. Total RNAs were extracted from each bulked sample using an RNeasy Plant Mini Kit (QIAGEN, Valencia, CA, USA). In the first RNA-Seq analysis, diverse sizes of flower buds larger than $3 \mathrm{~mm}$ were used. Flower buds smaller than $3 \mathrm{~mm}$ were included in the second analysis to cover early stages of male gametophyte development. The third analysis was performed using the TUMS4-S1 population after removing heterozygous MF individuals. Genotypes of heterozygous individuals were predicted using tightly linked molecular markers developed in this study.

RNA-Seq analyses were performed by a specialized company (Phyzen Genomics Institute, Seoul, Republic of Korea). Transcriptomes were sequenced using a HiSeq X Ten platform (Illumina, Hayward, CA, USA). Trimmed reads were separately mapped to two reference transcriptomes (Kim et al. 2015b; Sohn et al. 
2016). Unmapped reads were pooled and de novo assembled into contigs using Trinity software (Haas et al. 2013). Identification of single nucleotide polymorphisms (SNPs) between bulked RNAs and quantification of transcription levels of contigs were carried out using SAMTools (Li et al. 2009) and RSEM software (Li and Dewey 2011), respectively.

To estimate transcription levels of orf725, real-time RT-PCRs were performed. Total RNAs were extracted from flower buds of five MF and five MS plants of the TUMS4 population using an RNeasy Plant Mini Kit (QIAGEN). Total RNAs extracted in the previous study (Kim et al. 2015a) from MF and MS $F_{2}$ individuals with male-fertility phenotypes controlled by the Ms locus were also used for comparison of transcription levels. cDNAs were synthesized using a cDNA synthesis kit (SuperScript ${ }^{\text {TM }}$ III first-strand synthesis system for RT-PCR, Invitrogen, Carlsbad, CA, USA).

Real-time RT-PCRs were carried out using SYBR ${ }^{\circledR}$ Green Realtime PCR Master Mix (Toyobo Co. Ltd, Osaka, Japan) and a LightCycler ${ }^{\circledR} 96$ Real-Time PCR system (Roche Molecular Systems, Pleasanton, CA, USA) according to manufacturers' instructions with four technical replicates. cDNAs diluted 100-fold were then used as templates. Onion nad6 gene positioned in the master circle of mitochondrial genome (GenBank accession: KU318712) was used as an internal control. Primer sequences used for real-time RTPCR are shown in Supplementary Table 2.

\section{DNA extraction, PCR amplification, and high-resolution melting (HRM) marker analysis}

Total genomic DNAs of segregating populations were extracted from leaves or flower peduncle tissues using the cetyltrimethylammonium bromide (CTAB) method (Doyle and Doyle 1987). For sequencing of contigs, PCR amplifications were performed in a $25 \mu \mathrm{L}$ reaction mixture containing $0.1 \mu \mathrm{g}$ template, 2.5 $\mu \mathrm{L}$ 10x PCR buffer, $0.5 \mu \mathrm{L}$ forward primer $(10 \mu \mathrm{M}), 0.5 \mu \mathrm{L}$ reverse primer $(10 \mu \mathrm{M}), 0.5 \mu \mathrm{L}$ dNTPs $(10 \mathrm{mM}$ each), and $0.25 \mu \mathrm{L}$ polymerase mix (Advantage 2 Polymerase Mix, Takara Bio, Shiga, Japan). PCR amplification consisted of an initial denaturation step at $95^{\circ} \mathrm{C}$ for $4 \mathrm{~min}, 10$ cycles at $95^{\circ} \mathrm{C}$ for $30 \mathrm{~s}, 65^{\circ} \mathrm{C}$ $\left(0.8^{\circ} \mathrm{C}\right.$ decrements in each cycle) for $30 \mathrm{~s}$, and $72^{\circ} \mathrm{C}$ for $1 \mathrm{~min}, 35$ cycles at $95^{\circ} \mathrm{C}$ for $30 \mathrm{~s}, 57^{\circ} \mathrm{C}$ for $30 \mathrm{~s}$, and $72^{\circ} \mathrm{C}$ for $1 \mathrm{~min}$, and a final 10 min extension at $72^{\circ} \mathrm{C}$. After confirming successful PCR amplification by visualizing on $1.5 \%$ agarose gels after ethidium bromide staining, PCR products were purified for sequencing using a QIAquick PCR Purification kit (QIAGEN). Sequencing was performed by a specialized company (Macrogen, Seoul, Republic of Korea). Regarding HRM and cleaved amplified polymorphic sequence (CAPS) markers, detail procedures were described in previous studies (Kim and Kim 2019; Cho et al. 2021). Primer sequences of molecular markers are shown in Supplementary Table 2.

\section{Results}

Identification of a novel locus responsible for fertility restoration of male-sterility caused by CMS-T cytoplasm in onions

An unstable MS phenotype producing a small amount of viable pollen grains was identified from openpollinated (OP) populations derived from PI273626 in a previous study (Kim et al. 2019a), although Ms 
genotypes of unstable MS plants were homozygous recessive. These OP populations and PI273626 contained the CMS-Y cytoplasm designated by Kim et al. (2019a). To investigate whether such an unstable MS phenotype occurred in other types of cytoplasm, two plants (Plants \#4 and \#9) showing unstable MS phenotypes were crossed with stable MS onions containing the CMS-T cytoplasm (Supplementary Fig. 1).

MF and MS plants segregated at a ratio of one to one in progeny populations (TUMS4 and TUMS9) of these two crosses (Table 1). In the case of MF onions (Figs. 1A and 1C), male-fertility phenotypes were completely normal with plenty of pollen grains. However, no pollen was detected in MS plants (Figs. 1B and 1D). Pollen shapes of MF plants were also normal with few deformed pollen grains (Supplementary Fig. 2), whereas many deformed pollen grains were observed in unstable MS plants (Kim et al. 2019a). Genotypes of six molecular markers showed almost perfect linkage disequilibrium with the Ms locus, confirming that genotypes of both MF and MS plants were all homozygous recessive for the Ms locus (Supplementary Fig. 3). These results indicated that male-fertility of these populations was restored by another novel Rf locus, not the Ms locus. Single-gene inheritance patterns of male-fertility restoration were also observed in successive generations (Table 1). This novel Rf locus identified in this study is designated as Ms2.

Table 1

Inheritance patterns of male-fertility phenotypes controlled by the Ms2 locus in segregating populations

\begin{tabular}{|c|c|c|c|c|c|c|}
\hline \multirow[t]{2}{*}{ Population $^{a}$} & \multirow[t]{2}{*}{ Expected genotype } & \multicolumn{2}{|c|}{ Male-fertility phenotype ${ }^{b}$} & \multirow[t]{2}{*}{ Total } & \multirow[t]{2}{*}{$x^{2}$} & \multirow[t]{2}{*}{$P$} \\
\hline & & MF & MS & & & \\
\hline TUMS4 & $m s 2 m s 2 \otimes M s 2 m s 2$ & 20 & 19 & 39 & 0.026 & 0.87 \\
\hline TUMS9 & $m s 2 m s 2 \otimes M s 2 m s 2$ & 104 & 114 & 218 & 0.46 & 0.50 \\
\hline TUMS4-S1 & $M s 2 m s 2$ selfing & 164 & 64 & 228 & 1.15 & 0.28 \\
\hline TUMS4-S3 & $M s 2 m s 2$ selfing & 309 & 98 & 407 & 0.18 & 0.67 \\
\hline TUMS4-C1 & $m s 2 m s 2 \otimes M s 2 m s 2$ & 148 & 157 & 305 & 0.27 & 0.61 \\
\hline TUMS4-C9 & $m s 2 m s 2 \otimes M s 2 m s 2$ & 204 & 200 & 404 & 0.040 & 0.84 \\
\hline \multicolumn{7}{|c|}{ 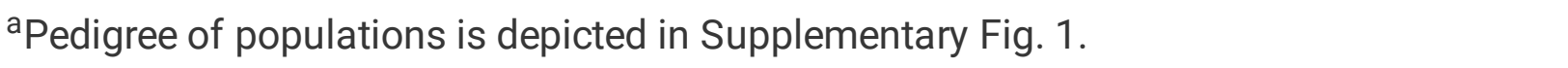 } \\
\hline
\end{tabular}

Identification of candidate genes responsible for the Ms2 locus and development of molecular markers tightly linked to the Ms2 locus 
To identify candidate genes responsible for the Ms2 locus, combined analyses of BSA and RNA-seq were carried out using bulked RNAs of MF and MS plants of the TUMS4 population. RNA-seq analyses were performed twice using the same population for repetition. Approximately $6 \mathrm{~Gb}$ raw reads were produced from each bulked RNA. Sequences of raw reads were deposited to the SRA database (Supplementary Table 3). After trimming low quality sequences, trimmed reads were mapped to the reference transcriptome (Supplementary Table 4). Approximately $80 \%$ trimmed reads were mapped to the reference transcriptome (Supplementary Table 5).

Using a customized screening process (Supplementary Table 6), 60 contigs containing SNPs between MF and MS bulked RNAs were selected after visual investigation of SNPs using an IGV viewer software (Robinson et al. 2011). Among these contigs, one contig (AC.Combine.Locus_14688.6) was mapped to the end of chromosome 2 in a previous study (Cho et al. 2021). In addition, four contigs showed high homologies with corresponding contigs located at the chromosome 2 of another linkage map (Supplementary Table 7). These results suggested that the Ms2 locus was likely to be positioned at the end of chromosome 2. Eight HRM markers were developed to construct an Ms2-flanking linkage map using contigs screened in this study or contigs showing high homologies with the loci mapped by Duangjit et al. (2013). Since the Ms locus was also positioned at chromosome 2, previously developed markers linked to the $M s$ were included to compare their chromosomal positions. Linkage maps indicated that the Ms2 locus was positioned at the end of chromosome 2 and linked to the Ms locus with a distance of approximately $70 \mathrm{cM}$ (Fig. 2).

To identify more candidate genes, additional RNA-seq analysis was performed after removing heterozygous MF individuals from MF bulked RNA (Supplementary Table 3). Trimmed reads produced from three RNA-seq analyses were also mapped to another reference transcriptome (Sohn et al. 2016). Trimmed reads were also mapped to the de novo assembled transcriptome (Supplementary Tables 4 and 5). Pooled reads which were unmapped to the reference transcriptome (Kim et al. 2015b) from three RNASeq analyses were used for de novo assembly of contigs.

Using a less stringent screening process (Supplementary Table 8), contigs containing homozygous SNPs between bulked RNAs were selected. Among 164 screened contigs, 38 contigs in which SNPs were clearly supported by sufficient read depths were selected for developing HRM markers and identifying candidate genes (Supplementary Table 9). To determine linkage relationship of these selected contigs and the Ms2 locus, a total of 30 recombinants between $\mathrm{H} 89571$ and $\mathrm{H} 56318$ were identified from 1,344 individuals of four segregating populations (Supplementary Table 10). H53996 and H36898 markers flanked the Ms2 locus most tightly. However, no contig showing perfect linkage to the Ms2 locus was identified (Supplementary Table 10).

\section{Analysis of differentially expressed genes (DEGs) between bulked RNAs and effect of dominant Ms2 allele on transcription of orf 725}

Overall transcription levels of all contigs in three types of transcriptomes were similar between MF and MS bulked RNAs, showing correlation coefficients of more than 0.8 in all cases (Supplementary Fig. 4). 
Using a stepwise screening process (Supplementary Table 11), a total of 204 contigs were selected as DEGs between bulked RNAs (Supplementary Table 12). No plausible candidate genes such as PPRcoding genes were identified. Many contigs seemed to be involved in general processes of male gametophyte development. For this reason, the majority of DEGs selected in this study showed reduced transcription in MS bulked RNA previously isolated from a $F_{2}$ population (Kim et al. 2015a) in which male-fertility phenotypes were determined by the Ms locus (Fig. 3).

Interestingly, amounts of transcripts of orf725, the causal gene for CMS induction in onions, were reduced in MF bulked RNAs of all three RNA-Seq analyses, whereas a higher amount of orf725 transcripts was observed in MF bulked RNAs isolated from the $F_{2}$ population controlled by the Ms locus (Fig. 4A). Realtime RT-PCRs were carried out to confirm reduced transcription of orf725 in MF plants. Reduced transcripts of orf 725 were observed in all five MF individuals, while no significant difference was observed in $\mathrm{F}_{2}$ individuals in which male-fertility was controlled by the Ms locus (Figs. 4B and 4C). These results suggested that male-fertility might be restored by different mechanisms depending on Rf loci in onions.

\section{Discussion}

\section{Discovery of a novel $R f$ locus in onion and its implication in onion $F_{1}$ hybrid breeding}

A novel Rf locus was identified from OP populations derived from PI273626 in this study. Clear singlegene inheritance patterns and reliable positioning of the Ms2 locus at the end of chromosome 2 proved existence of a novel Rf locus in addition to the Ms locus. Based on complex segregation ratios, Schweisguth (1973) has suggested involvement of three independent Rf loci in fertility restoration of male-sterility conferred by CMS-T cytoplasm. However, this is the first report about the discovery of the second onion Rf locus with specific chromosomal position revealed by constructing a linkage map. Since genotypes of the Ms locus were fixed as homozygous recessive in all segregating populations used in this study and their genotypes could be confirmed by multiple molecular markers tagging the Ms locus (Supplementary Fig. 3), segregation of male-fertility controlled by the Ms2 locus could be successfully identified in this study.

Considering the fact that male-fertility phenotypes of numerous onion breeding lines in Korea and probably other countries could be perfectly predicted by molecular markers showing linkage disequilibrium with the Ms locus (Kim 2014; Kim et al. 2015a), existence of the Ms2 locus was somewhat unexpected. Since male-fertility phenotypes of the $\mathrm{S}_{1}$ population produced by self-pollination of PI273626 were perfectly matched with genotypes of molecular markers tagging the Ms locus and that unstable male-sterility was identified from a single plant in the $\mathrm{OP}_{1}$ population (Kim et al. 2019a), a dominant Ms2 allele might have been introduced from other unknown exotic germplasm.

Unlike breeding lines used in Korea, it was assumed that frequency of dominant Ms2 allele might be higher in exotic onion germplasm. Khar and Saini (2016) and Ferreira and Santos (2018) have reported 
that some populations show discrepancy between genotypes of molecular markers tagging the Ms locus and phenotypes of male-fertility in Indian and Brazilian onion germplasm, respectively. Such discrepancies observed in Indian and Brazilian accessions might be caused by presence of the Ms2 locus. Further studies are needed to clarify this. Considering the fact that unstable male-sterility was attributable to a relatively low expressivity of the Ms2 locus as observed in a previous study (Kim et al. 2019a), it is desirable to eradicate the dominant Ms2 allele among breeding lines for efficient deployment of marker-assisted selection of the Ms locus, the major Rf locus in onions. Molecular markers tagging the Ms2 locus developed in this study could be efficiently used for this purpose.

\section{Mechanism underlying male-fertility restoration by the Ms2 locus and factors affecting the function of Ms2}

Inheritance patterns of male-fertility in segregating populations showed that both $M s$ and $M s 2$ loci were redundantly involved in fertility restoration in onions. When genotypes of both Rf loci were homozygous recessive, stable male-sterility was observed, although the efficacy of the Ms locus might be higher than that of Ms2 locus. In a previous study (Kim et al. 2019a), stable male-fertility was observed when at least one dominant Ms allele was present. However, an unstable MS phenotype was detected when Ms and Ms2 genotypes were homozygous recessive and heterozygous, respectively.

Genetic redundancy is typically arisen by gene duplication. It is widespread in higher organisms (Pickett and Meeks-Wagner 1995; Nowak et al. 1997; Kafri et al. 2009). Whether causal genes for Ms and Ms2 loci are paralogs is not resolved yet. Mechanisms by which male-fertility is restored might be different from each other. Unlike the Ms locus which did not affect transcription of orf725, transcripts of orf 725 were significantly reduced in MF $\mathrm{F}_{2}$ individuals (Fig. 4). The causal gene for $\mathrm{Ms} 2 \mathrm{might}$ be able to restore malefertility by suppressing transcription of orf725 or degrading transcripts. Suppression of transcription of CMS-associated genes by Rf genes is frequently observed in other crops (Kennell and Pring 1989; Gagliardi and Leaver 1999; Menassa et al. 1999; Tang et al. 1999; Wang et al. 2006).

One interesting feature of male-fertility restored by the $M s 2$ locus was that the stability of male-fertility might be affected by cytoplasm types. Unstable male-sterility was observed in plants of $\mathrm{OP}_{1}$ and $\mathrm{OP}_{2}$ populations containing the CMS-Y cytoplasm. Many deformed pollen grains were observed in these unstable MS plants (Kim et al. 2019a). However, male-fertility of plants containing the CMS-T cytoplasm and homozygous recessive Ms genotype was stably restored by the Ms2 locus. Since male-sterility conferred by the CMS-S cytoplasm was known to be more stable and more commonly used in $\mathrm{F}_{1}$ hybrid breeding than CMS-T male-sterility (Havey 2000), the effect of the Ms2 locus on male-fertility phenotypes of plants containing CMS-S cytoplasm needs to be analyzed in the future. In addition, more diverse accessions need to be analyzed to elucidate whether stability of male-fertility is caused by types of cytoplasm or other nuclear gene(s).

In addition, effects of environmental factors cannot be excluded. In particular, temperature might affect the stability of male-fertility controlled by the Ms 2 locus. The effect of temperature on male-fertility 
phenotypes of onions has been previously reported (Barham and Munger 1950; van der Meer and van Bennekom 1969). Stability of male-sterility was high at a low temperature $\left(14^{\circ} \mathrm{C}\right)$. However, viable pollen grains appeared at a high temperature $\left(23^{\circ} \mathrm{C}\right)$. The degree of stability varied depending on populations. Male-sterility of two hybrids introduced from the United States was hardly affected by temperature in comparison to European accessions (van der Meer and van Bennekom 1969). Since vulnerability to temperature might vary depending on populations, genetic factors such as Ms2 locus might be related to temperature sensitivity of male-sterility in onions.

Effects of interaction between genetic factors and temperature on male-fertility have been reported in rice (Chen et al. 2007; Zhou et al. 2012) and wheat (Song et al. 2013). They have been utilized to implement a two-line hybrid system. Although most thermo-sensitive male-sterility are GMS, a thermo-sensitive CMS line has also been developed in wheat (Song et al. 2013). Similarly, if interaction between Ms2 and temperature was elucidated, it could be used to establish a two-line system in onions. However, it would be an ideal strategy to eradicate the dominant Ms2 allele from breeding populations since other unknown factors might also affect the stability of male-fertility controlled by the Ms2 locus. Indeed, seven plants showing unstable MF or MS phenotypes were observed in populations analyzed in this study. These plants showing unstable phenotypes were identified by discrepancy of phenotypes and genotypes of molecular markers tightly flanking the Ms2 locus (data not shown). Taken together, results presented in this study could be used to develop an optimal strategy for $F_{1}$ hybrid breeding of onions.

\section{Declarations}

\section{Acknowledgments}

The authors thank Ji-hwa Heo, Jeong-An Yoo, and Su-jeong Kim for their dedicated technical assistance.

\section{Authors' contribution}

Nari Yu performed experiments and drafted the manuscript. Sunggil Kim organized and coordinated this research project and edited the final manuscript.

\section{Funding}

This study was supported by the Korea Institute of Planning and Evaluation for Technology in Food, Agriculture and Forestry (IPET) via Golden Seed Project (Center for Horticultural Seed Development, No 213007-05-5-SBB10).

\section{Ethics approval}

All experiments were performed in compliance with current laws of the Republic of Korea.

\section{Consent for publication}


Not applicable.

\section{Competing interests}

The authors declare no competing interest.

\section{References}

1. Abdelnoor RV, Yule R, Elo A, Christensen AC, Meyer-Gauen G, Mackenzie SA (2003) Substoichiometric shifting in the plant mitochondrial genome is influenced by a gene homologous to MutS. Proc Natl Acad Sci USA 100:5968-5973

2. Albert B, Godelle B, Gouyon PH (1998) Evolution of the plant mitochondrial genome: dynamics of duplication and deletion of sequences. J Mol Evol 46:155-158

3. Allen JO, Fauron CM, Mink P, Roark L, Oddiraju S, Lin GN, Meyer L, Sun H, Kim K, Wang C, Du F, Xu D, Gibson M, Cifrese J, Clifton SW, Newton KJ (2007) Comparisons among two fertile and three malesterile mitochondrial genomes of maize. Genetics 177:1173-1192

4. Arrieta-Montiel M, Lyznik A, Woloszynska M, Janska H, Tohme J, Mackenzie SA (2001) Tracing evolutionary and developmental implications of mitochondrial stoichiometric shifting in the common bean. Genetics 158:851-864

5. Backert S, Neilsen BL, Börner T (1997) The mystery of the rings: structure and replication of mitochondrial genomes from higher plants. Trends Plant Sci 2:477-483

6. Bang H, Kim S, Park SO, Yoo K, Patil BS (2013) Development of a codominant CAPS marker linked to the Ms locus controlling fertility restoration in onion (Allium cepa L.). Sci Hortic 153:42-49

7. Barham WS, Munger HM (1950) The stability of male sterility in onions. Proc Am Soc Hort Sci $56: 401-409$

8. Barkan A, Small I (2014) Pentatricopeptide repeat proteins in plants. Annu Rev Plant Biol 65:415-442

9. Bellaoui M, Martin-Canadell A, Pelletier G, Budar F (1998) Low-copy-number molecules are produced by recombination, actively maintained and can be amplified in the mitochondrial genome of Brassicaceae: relationship to reversion of the male sterile phenotype in some cybrids. Mol Gen Genet 257:177-185

10. Berninger $E$ (1965) Contribution à l'étude de la sterilité mâle de l'oignon (Allium cepa L.). Ann Amélior Plant 15:183-199

11. Bohra A, Jha UC, Adhimoolam P, Bisht D, Singh NP (2016) Cytoplasmic male sterility (CMS) in hybrid breeding in field crops. Plant Cell Rep 35:967-993

12. Chen L, Liu Y (2013) Male sterility and fertility restoration in crops. Ann Rev Plant Biol 65:579-606

13. Chen R, Zhao N, Li S, Grover CE, Nie H, Wendel JF, Hua J (2017) Plant mitochondrial genome evolution and cytoplasmic male sterility. Crit Rev Plant Sci 36:55-69

14. Chen R, Zhao X, Shao Z, Wei Z, Wang Y, Zhu L, Zhao J, Sun M, He R, He G (2007) Rice UDP-glucose pyrophosphorylase 1 is essential for pollen callose deposition and its cosuppression results in a new 
type of thermosensitive genic male sterility. Plant Cell 19:847-861

15. Cho Y, Kim B, Lee J, Kim S (2021) Construction of a high-resolution linkage map and chromosomal localization of the loci determining major qualitative traits in onion (Allium cepa L.). Euphytica $217: 17$

16. Cui X, Wise RP, Schnable PS (1996) The rf2 nuclear restorer gene of male-sterile T-cytoplasm maize. Science 272:1334-1336

17. Doyle JJ, Doyle JL (1987) A rapid DNA isolation procedure for small quantities of fresh leaf tissue. Phytochem Bull 19:11-15

18. Duangjit J, Bohanec B, Chan AP, Town CD, Havey MJ (2013) Transcriptome sequencing to produce SNP-based genetic maps of onion. Theor Appl Genet 126:2093-2101

19. Fauron CMR, Moore B, Casper M (1995) Maize as a model of higher plant mitochondrial genome plasticity. Plant Sci 112:11-32

20. Ferreira RR, Santos CAF (2018) Partial success of marker-assisted selection of 'A' and 'B' onion lines in Brazilian germplasm. Sci Hortic 242:110-115

21. Fujii S, Toriyama K (2009) Suppressed expression of RETROGRADE-REGULATED MALE STERILITY restores pollen fertility in cytoplasmic male sterile rice plants. Proc Natl Acad Sci USA 106:9513-9518

22. Gaborieau L, Brown GG, Mireau H (2016) The propensity of pentatricopeptide repeat genes to evolve into restorers of cytoplasmic male sterility. Front Plant Sci 7:1816

23. Gagliardi D, Leaver CJ (1999) Polyadenylation accelerates the degradation of the mitochondrial mRNA associated with cytoplasmic male sterility in sunflower. EMBO J 18:3757-3766

24. Gökçe AF, Havey MJ (2002) Linkage equilibrium among tightly linked RFLPs and the Ms locus in open-pollinated onion populations. J Amer Soc Hort Sci 127:944-946

25. Haas BJ, Papanicolaou A, Yassour M, Grabherr M, Blood PD, Bowden J, Couger MB, Eccles D, Li B, Lieber M, Macmanes MD, Ott M, Orvis J, Pochet N, Strozzi F, Weeks N, Westerman R, William T, Dewey CN, Henschel R, Leduc RD, Friedman N, Regev A (2013) De novo transcript sequence reconstruction from RNA-seq using the Trinity platform for reference generation and analysis. Nat Protoc 8:1494512

26. Hanson MR, Bentolila S (2004) Interactions of mitochondrial and nuclear genes that affect male gametophyte development. Plant Cell 16:S154-S169

27. Havey MJ (2000) Diversity among male-sterility-inducing and male-fertile cytoplasms of onion. Theor Appl Genet 101:778-782

28. Havey MJ (2013) Single nucleotide polymorphisms in linkage disequilibrium with the male-sterility restoration (Ms) locus in open-pollinated and inbred populations of onion. J Amer Soc Hort Sci 138:306-309

29. Hu J, Wang K, Huang W, Liu G, Gao Y, Wang J, Huang Q, Ji Y, Qin X, Wan L, Zhu R, Li S, Yang D, Zhu Y (2012) The rice pentatricopeptide repeat protein RF5 restores fertility in Hong-Lian cytoplasmic malesterile lines via a complex with the glycine-rich protein GRP162. Plant Cell 24:109-122 
30. Huo YM, Liu BJ, Yang YY, Miao J, Gao LM, Kong SP, Wang ZB, Kitano H, Wu X (2015) AcSKP1, a multiplex PCR-based co-dominant marker in complete linkage disequilibrium with the male-fertility restoration (Ms) locus, and its application in open-pollinated populations of onion. Euphytica 204:711-722

31. Janska H, Sarria R, Woloszynska M, Arrieta-Montiel M, Mackenzie SA (1998) Stoichiometric shifts in the common bean mitochondrial genome leading to male sterility and spontaneous reversion to fertility. Plant Cell 10:1163-1180

32. Jones HA, Clarke A (1943) Inheritance of male sterility in the onion and the production of hybrid seed. Proc Am Soc Hort Sci 43:189-194

33. Jones HA, Emsweller SL (1936) A male-sterile onion. Proc Am Soc Hort Sci 34:582-585

34. Kafri R, Springer M, Pilpel Y (2009) Genetic redundancy: New tricks for old genes. Cell 136:389-392

35. Kanazawa A, Tsutsumi N, Hirai A (1994) Reversible changes in the composition of the population of mtDNAs during dedifferentiation and regeneration in tobacco. Genetics 138:865-870

36. Kennell JC, Pring DR (1989) Initiation and processing of atp6, T-urf13 and ORF221 transcripts from mitochondria of T cytoplasm maize. Mol Gen Genet 216:16-24

37. Khar A, Saini N (2016) Limitations of PCR-based molecular markers to identify male-sterile and maintainer plants from Indian onion (Allium cepa L.) populations. Plant Breed 135:519-524

38. Kim B, Kim C, Kim S (2019a) Inheritance of fertility restoration of male-sterility conferred by cytotype $\mathrm{Y}$ and identification of instability of male fertility phenotypes in onion (Allium cepa L.). J Hort Sci Biotechnol 94:341-348

39. Kim B, Kim K, Yang T, Kim S (2016) Completion of the mitochondrial genome sequence of onion (Allium cepa L.) containing the CMS-S male-sterile cytoplasm and identification of an independent event of the $c c m F_{N}$ gene split. Curr Genet 62:873-885

40. Kim B, Kim S (2019) Identification of a variant of CMS-T cytoplasm and development of high resolution melting markers for distinguishing cytoplasm types and genotyping a restorer-of-fertility locus in onion (Allium cepa L.). Euphytica 215:164

41. Kim B, Yang T, Kim S (2019b) Identification of a gene responsible for cytoplasmic male-sterility in onions (Allium cepa L.) using comparative analysis of mitochondrial genome sequences of two recently diverged cytoplasms. Theor Appl Genet 132:313-322

42. Kim S (2014) A codominant molecular marker in linkage disequilibrium with a restorer-of-fertility gene $(M s)$ and its application in reevaluation of inheritance of fertility restoration in onions. Mol Breed 34:769-778

43. Kim S, Kim C, Park M, Choi D (2015a) Identification of candidate genes associated with fertility restoration of cytoplasmic male-sterility in onion (Allium cepa L.) using a combination of bulked segregant analysis and RNA-seq. Theor Apple Genet 128:2289-2299

44. Kim S, Kim M, Kim Y, Yeom S, Cheong K, Kim K, Jeon J, Kim S, Kim D, Sohn S, Lee Y, Choi D (2015b) Integrative structural annotation of de novo RNA-Seq provides an accurate reference gene set of the 
enormous genome of the onion (Allium cepa L.). DNA Res 22:19-27

45. Kim S, Lee E, Cho DY, Han T, Bang H, Patil BS, Ahn YK, Yoon M (2009) Identification of a novel chimeric gene, orf725, and its use in development of a molecular marker for distinguishing three cytoplasm types in onion (Allium cepa L.). Theor Appl Genet 118:433-441

46. Kitazaki K, Arakawa T, Matsunaga M, Yui-Kurino R, Matsuhira H, Mikami T, Kubo T (2015) Posttranslational mechanisms are associated with fertility restoration of cytoplasmic male sterility in sugar beet (Beta vulgaris). Plant J 83:290-299

47. Kitazaki K, Kubo T (2010) Cost of having the largest mitochondrial genome: Evolutionary mechanism of plant mitochondrial genome. J Bot 2010:620137

48. Kmiec B, Woloszynska M, Janska $\mathrm{H}$ (2006) Heteroplasmy as a common state of mitochondrial genetic information in plants and animals. Curr Genet 50:149-159

49. Li B, Dewey CN (2011) RSEM: accurate transcript quantification from RNA-Seq data with or without a reference genome. BMC Bioinformatics 12:323

50. Li H, Handsaker B, Wysoker A, Fennell T, Ruan J, Homer N, Marth G, Abecasis G, Durbin R and 1000 Genome Project Data Processing Subgroup (2009) The Sequence alignment/map (SAM) format and SAMtools. Bioinformatics 25:2078-2079

51. Manna S (2015) An overview of pentatricopeptide repeat proteins and their applications. Biochimie 113:93-99

52. McCauley DE, Bailey MF (2009) Recent advances in the study of gynodioecy: the interface of theory and empiricism. Ann Bot 104:611-620

53. Menassa R, L'Homme Y, Brown GG (1999) Post-transcriptional and developmental regulation of a CMS-associated mitochondrial gene region by a nuclear restorer gene. Plant J 17:491-499

54. Michelmore RW, Paran I, Kesseli RV (1991) Identification of markers linked to disease-resistance genes by bulked segregant analysis: A rapid method to detect markers in specific genomic regions by using segregating populations. Proc Natl Acad Sci USA 88:9828-9832

55. Nowak MA, Boerlijst MC, Cooke J, Smith JM (1997) Evolution of genetic redundancy. Nature 388:167-171

56. Oldenburg DJ, Bendich AJ (2001) Mitochondrial DNA from the Liverwort Marchantia polymorpha: Circularly permuted linear molecules, head-to-tail concatemers, and a 5' protein. J Mol Biol 310:549562

57. Park J, Bang H, Cho DY, Yoon M, Patil BS, Kim S (2013) Construction of high-resolution linkage map of the Ms locus, a restorer-of-fertility gene in onion (Allium cepa L.). Euphytica 192:267-278

58. Pickett FB, Meeks-Wagner DR (1995) Seeing double: appreciating genetic redundancy. Plant Cell 7:1347-1356

59. Robinson JT, Thorvaldsdóttir H, Winckler W, Guttman M, Lander ES, Getz G, Mesirov JP (2011) Integrative genomics viewer. Nat Biotechnol 29:24-26 
60. Sakai T, Imamura J (1993) Evidence for a mitochondrial sub-genome containing radish AtpA in a Brassica napus cybrid. Plant Sci 90:95-103

61. Sandhu AP, Abdelnoor RV, Mackenzie SA (2007) Transgenic induction of mitochondrial rearrangements for cytoplasmic male sterility in crop plants. Proc Natl Acad Sci USA 104:1766-1770

62. Satoh M, Kubo T, Nishizawa S, Estiati A, Itchoda N, Mikami T (2004) The cytoplasmic male-sterile type and normal type mitochondrial genomes of sugar beet share the same complement of genes of known function but differ in the content of expressed ORFs. Mol Genet Genomics 272:247-256

63. Schweisguth B (1973) Étude d'un nouveau type de stérilité male chez l'oignon, Allium cepa L. Ann Amélior Plant 23:221-233

64. Shedge V, Arrieta-Montiel M, Christensen AC, Mackenzie SA (2007) Plant mitochondrial recombination surveillance requires unusual $\operatorname{Rec} A$ and MutS homologs. Plant Cell 19:1251-1264

65. Skippington E, Barkman TJ, Rice DW, Palmer JD (2015) Miniaturized mitogenome of the parasitic plant Viscum scurruloideum is extremely divergent and dynamic and has lost all nad genes. Proc Natl Acad Sci USA 112:E3515-3524

66. Sloan DB (2013) One ring to rule them all? Genome sequencing provides new insights into the 'master circle' model of plant mitochondrial DNA structure. New Phytol 200:978-985

67. Sloan DB, Alverson AJ, Chuckalovcak JP, Wu M, McCauley DE, Palmer JD, Taylor DR (2012) Rapid evolution of enormous, multichromosomal genomes in flowering plant mitochondria with exceptionally high mutation rates. PLoS Biol 10:e1001241

68. Sloan DB, Alverson AJ, Storchová H, Palmer JD, Taylor DR (2010) Extensive loss of translational genes in the structurally dynamic mitochondrial genome of the angiosperm Silene latifolia. BMC Evol Biol 10:274

69. Small I, Suffolk R, Leaver CJ (1989) Evolution of plant mitochondrial genomes via substoichiometric intermediates. Cell 58:69-76

70. Sohn S, Ahn Y, Lee T, Lee J, Jeong M, Seo C, Chandra R, Kwon Y, Kim C, Kim D, Won S, Kim JS, Choi D (2016) Construction of a draft reference transcripts of onion (Allium cepa) using long-read sequencing. Plant Biotechnol Rep 10:383-390

71. Song XY, Zhang LL, Zeng JL, Qian HH, Li HB, He BR (2013) Development of thermo-sensitive cytoplasmic male sterile (TCMS) lines of wheat characterized by complete male sterility at lowertemperatures and partially restored fertility at higher-temperatures. Euphytica 192:393-399

72. Tang HV, Chen W, Pring DR (1999) Mitochondrial orf107transcription, editing, and nucleolytic cleavage conferred by the gene Rf3 are expressed in sorghum pollen. Sex Plant Reprod 12:53-59

73. Van der Meer QP, van Bennekom JL (1969) Effect of temperature on the occurrence of male sterility in onion (Allium cepa L.). Euphytica 18:389-394

74. Wan X, Wu S, Li Z, Dong Z, An X, Ma B, Tian Y, Li J (2019) Maize genic male-sterility genes and their applications in hybrid breeding: progress and perspectives. Mol Plant 12:321-342 
75. Wang Z, Zou Y, Li X, Zhang Q, Chen L, Wu H, Su D, Chen Y, Guo J, Luo D, Long Y, Zhong Y, Liu Y (2006) Cytoplasmic male sterility of rice with Boro II cytoplasm is caused by a cytotoxic peptide and is restored by two related PPR motif genes via distinct modes of mRNA silencing. Plant Cell 18:676687

76. Woloszynska M, Trojanowski D (2009) Counting mtDNA molecules in Phaseolus vulgaaris: sublimons are constantly produced by recombination via short repeats and undergo rigorous selection during substoichiometric shifting. Plant Mol Biol 70:511-521

77. Yang YY, Huo YM, Miao J, Liu BJ, Kong SP, Gao LM, Liu C, Wang ZB, Tahara Y, Kitano H, Wu X (2013) Identification of two SCAR markers co-segregated with the dominant $M s$ and recessive $m s$ alleles in onion (Allium cepa L.). Euphytica 190:267-277

78. Zaegel V, Guermann B, Le Ret M, Andrés C, Meyer D, Erhardt M, Canaday J, Gualberto JM, Imbault P (2006) The plant-specific ssDNA binding protein OSB1 is involved in the stoichiometric transmission of mitochondrial DNA in Arabidopsis. Plant Cell 18:3548-3563

79. Zhou H, Liu Q, Li J, Jiang D, Zhou L, Wu P, Lu S, Li F, Zhu L, Liu Z, Chen L, Liu Y, Zhuang C (2012) Photoperiod- and thermo-sensitive genic male sterility in rice are caused by a point mutation in a novel noncoding RNA that produces a small RNA. Cell Res 22:649-660

\section{Figures}



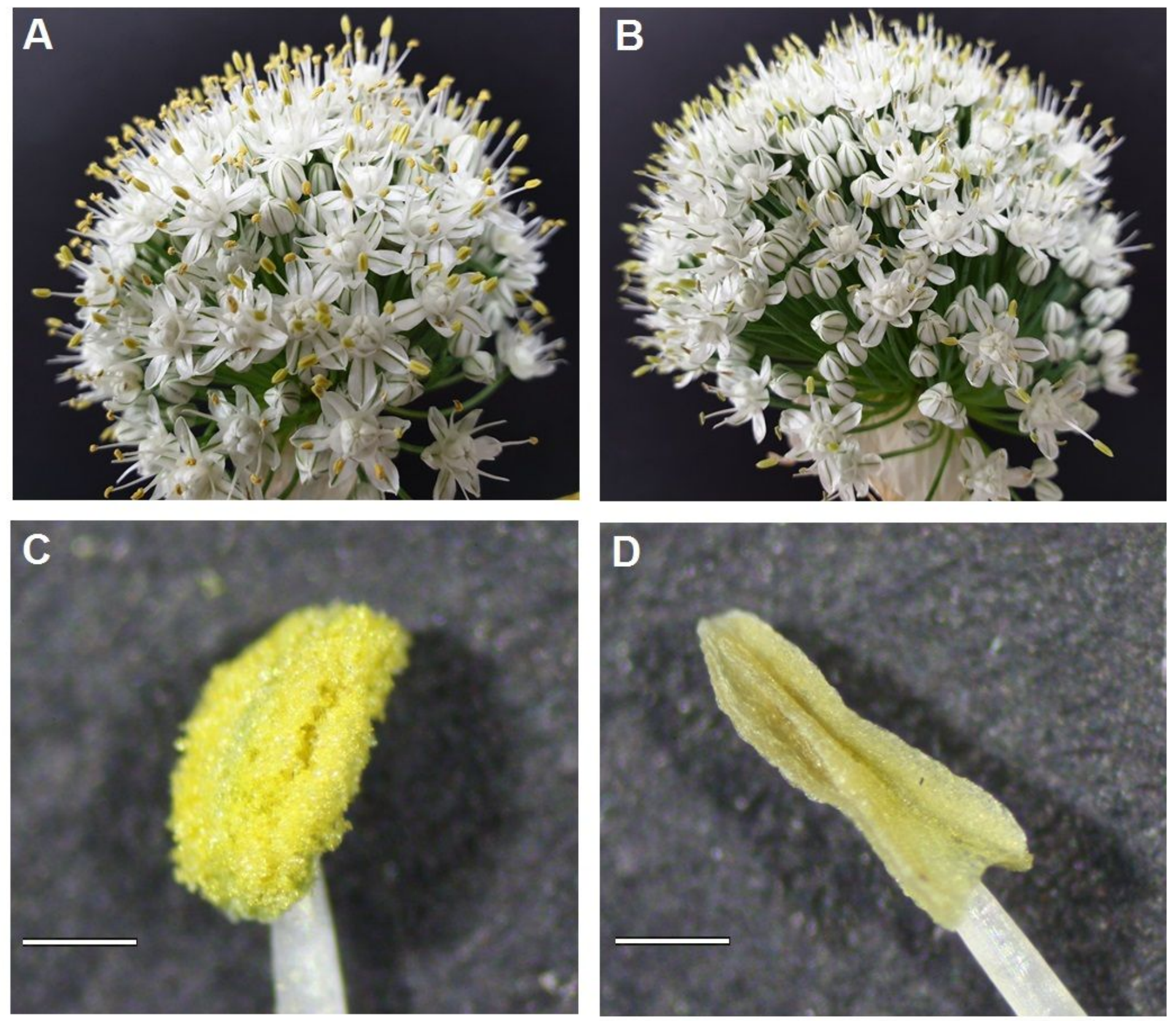

\section{Figure 1}

Morphologies of umbels and anthers of male-fertile and male-sterile onions in the TUMS4 population. A. Male-fertile umbel, B. Male-sterile umbel, C. Male-fertile anther, D. Male-sterile anther. Scale bars in images of anthers indicate $0.5 \mathrm{~mm}$. 

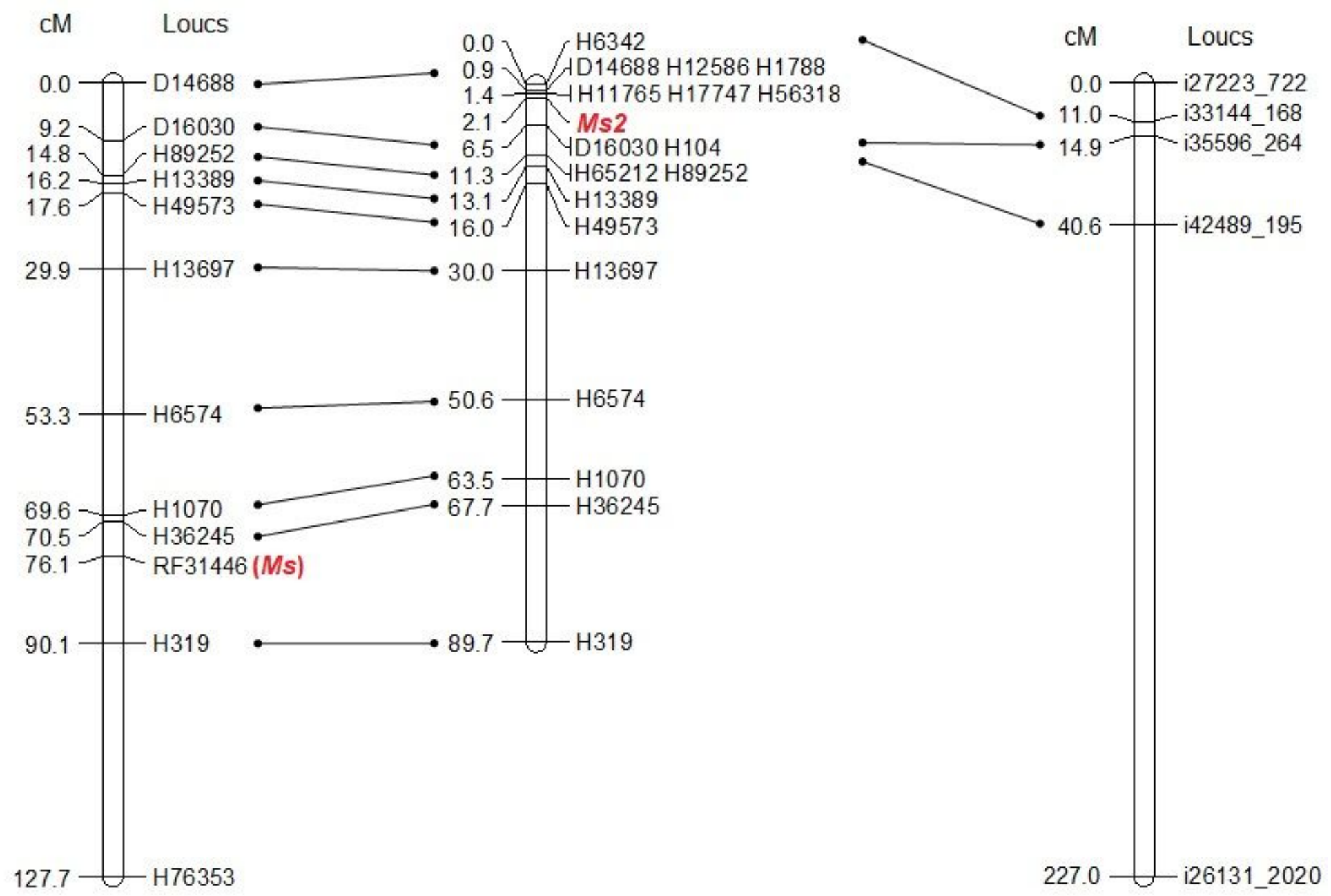

Figure 2

Onion linkage maps flanking the Ms2 locus. Left: Linkage map of chromosome 2 constructed by Cho et al. (2021); Center: Ms2-flanking linkage map constructed in this study; Right: Linkage map of chromosome 2 constructed by Duangjit et al. (2013). Homologous and identical contigs or markers are connected with horizontal lines. Ms and Ms2 loci are shown as red boldfaces. 

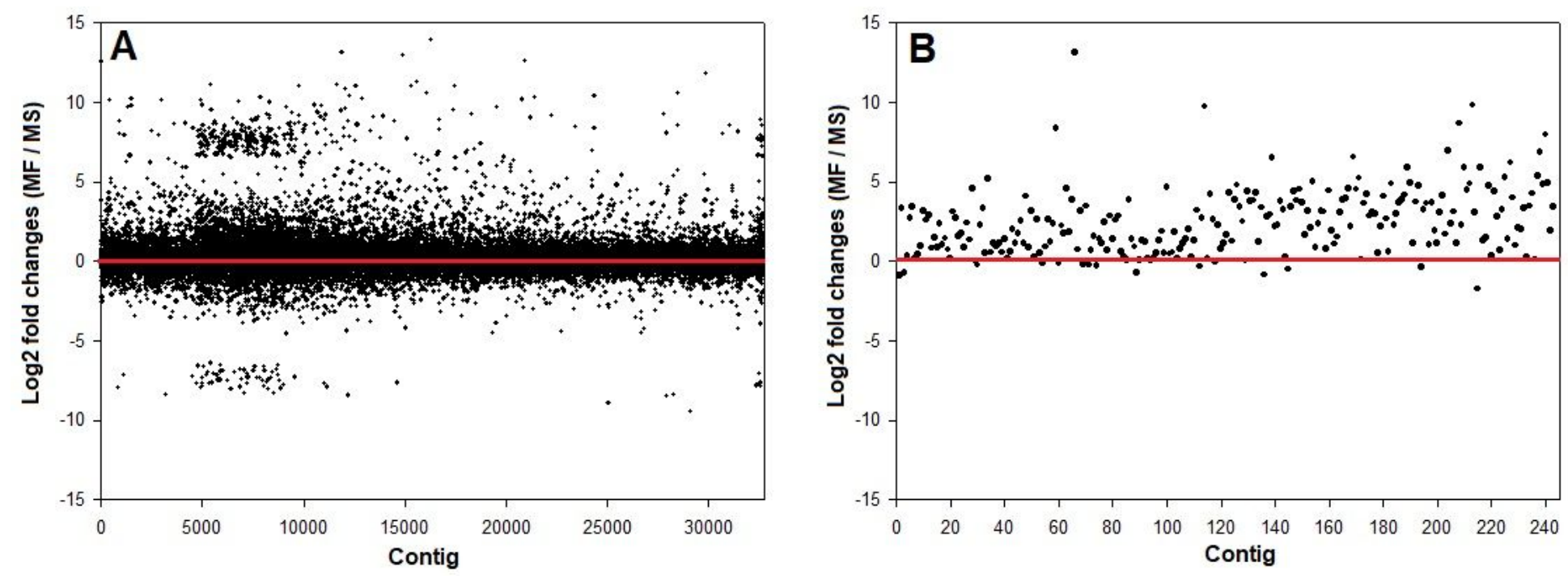

Figure 3

Fold changes of transcription levels of contigs between male-fertile and male-sterile bulked RNAs isolated in a previous study (Kim et al. 2015a) from the F2 population in which male-fertility phenotypes were controlled by the Ms locus. A. Fold changes of all contigs. These contigs were de novo assembled by Kim et al. (2015a). B. Fold changes of selected contigs showing high homologies with DEGs selected in this study (Supplementary Table 12). Red lines indicate equal levels of transcription between male-fertile and male-sterile bulked RNAs. 

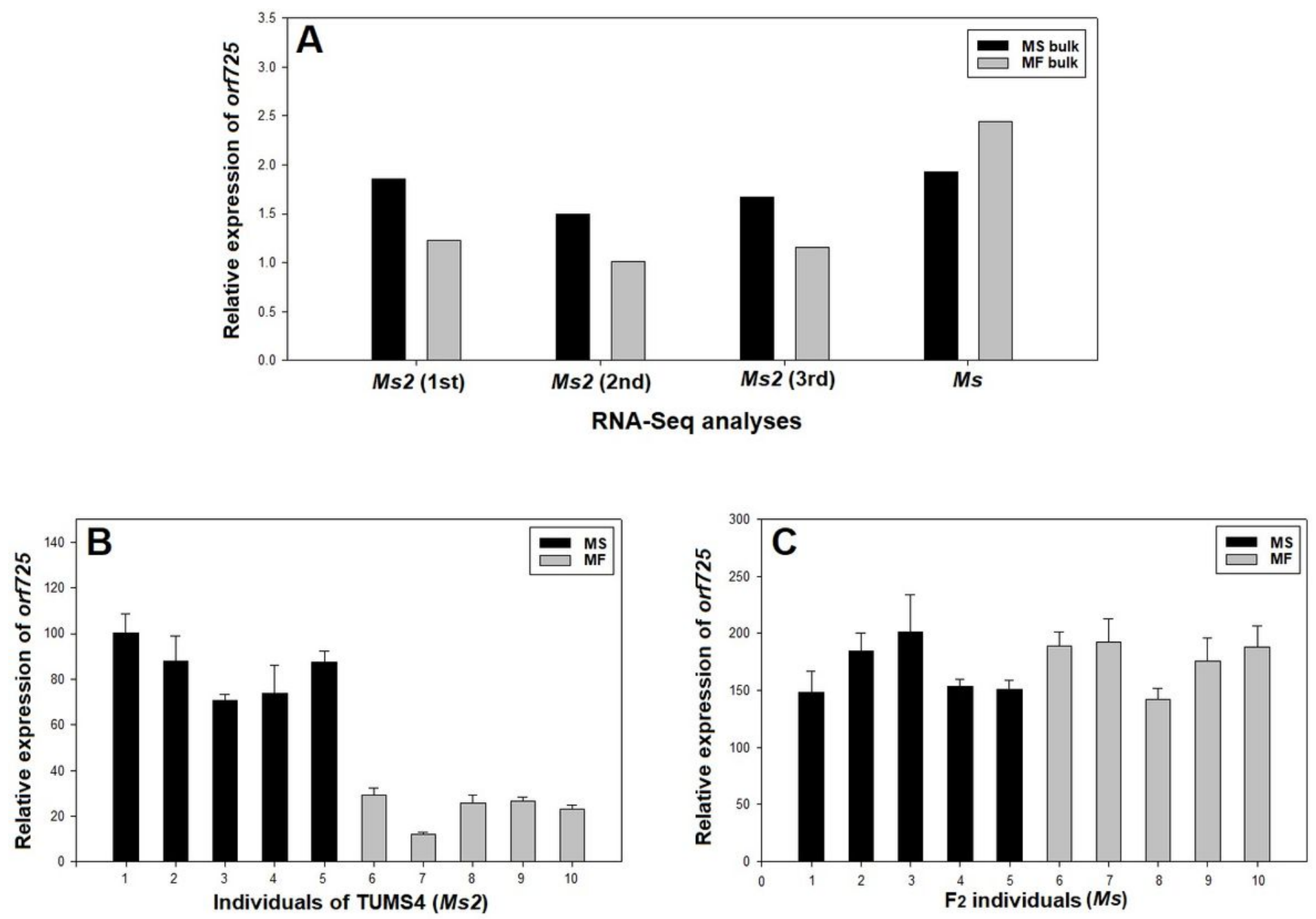

Figure 4

Comparison of relative expression levels of orf725 between male-fertile and male-sterile individuals of populations in which male-fertility phenotypes were determined by Ms and Ms2 loci, respectively.

Expression levels of nad6, a mitochondrial gene, were used as internal controls. A. Relative expression of orf725 estimated using data from RNA-Seq analyses. B. Relative expression levels of orf725 in individuals of the TUMS4 population. C. Relative expression levels of orf725 in F2 individuals of the population produced in a previous study (Kim et al. 2015a).

\section{Supplementary Files}

This is a list of supplementary files associated with this preprint. Click to download.

- SupplementaryFig.1.Pedigree.tif

- SupplementaryFig.2.SEMofpollengrains.tif

- SupplementaryFig.3.GenotypesofMslinkedmarkers.tif

- SupplementaryFig.4.Correlationofexpressionlevels.tif 
- SupplementaryTable1.Statisticsoftemperatures.xlsx

- SupplementaryTable2.Primersequenceusedinthisstudy.xlsx

- SupplementaryTable3.SummaryofRNASeq.xIsx

- SupplementaryTable4.Transcriptomeinformation.docx

- SupplementaryTable5.Readmappingtotranscriptomes.xlsx

- SupplementaryTable6.ScreeningprocessofSNPs.docx

- SupplementaryTable7.Listofhomologouscontigs.docx

- SupplementaryTable8.ScreeningprocessofhomozygousSNPs.docx

- SupplementaryTable9.ListofcontigscontainingSNPs.xIsx

- SupplementaryTable10.Recombinantanalysis.xlsx

- SupplementaryTable11.ScreeningprocessofDEGs.docx

- SupplementaryTable12.ListofDEGs.xlsx 